\title{
The Effect of Sunscreen on Skin Elastase Activity Induced by Ultraviolet-A Irradiation
}

\author{
Kazue Tsukahara, ${ }^{*, a}$ Shigeru Moriwaki, ${ }^{a}$ Mitsuyuki Hotta, ${ }^{a}$ Tsutomu Fujimura, ${ }^{a}$ \\ Yoriko SugiYama-Nakagiri, ${ }^{a}$ Satoshi Sugawara, ${ }^{b}$ Takashi Kitahara, ${ }^{a}$ and Yoshinori Takema ${ }^{a}$ \\ ${ }^{a}$ Biological Science Laboratories, Kao Corporation; 2606 Akabane, Ichikai, Haga, Tochigi 321-3497, Japan: and ${ }^{b}$ Skin \\ Care Research Laboratories, Kao Corporation; 2-1-3 Bunka, Sumida-ku, Tokyo 131-8501, Japan. \\ Received June 23, 2005; accepted September 30, 2005
}

It has been reported that application of sunscreens prevents the photoaging of skin in animal models and in humans. We irradiated the dorsal skin of hairless mice with ultraviolet-A (UVA), and investigated the effects of sunscreens on skin elastase activity and on skin properties. Six-week-old female HR/ICR hairless mice were used in these experiments. After being treated with either a UVA sunscreen (also containing ultraviolet-B (UVB) sunscreen to eliminate any slight UVB in the UVA lamps; Protection Factor of UVA (PFA)=6, Sun Protection Factor $(\mathrm{SPF})=20$ ) or a vehicle, the dorsal skins of mice were irradiated with the UVA lamps at $22.3 \mathrm{~J} / \mathrm{cm}^{2} / \mathrm{d}, 5$ times a week. At the end of 15 weeks skin properties were evaluated and elastase activities were measured. In the vehicle control group, UVA irradiation increased the brightness and yellowing of the skin, decreased the water content of the stratum corneum, increased skin thickness, decreased skin elasticity, increased skin elastase activity, and decreased the ability of the skin to recover in a pinch test, as compared to an unirradiated group. All these differences were statistically significant. In the UVA sunscreen group, both the UVA induced skin damage and the increase in skin elastase activity were significantly inhibited, as compared to the vehicle group. However, as compared to the unirradiated group, skin elastase activity was significantly increased and immediate extensibility of skin (Ue) was significantly decreased, thereby indicating that the UVA sunscreen did not prevent photoaging to the same level as the unirradiated group. These results suggest the partial efficacy of the topical photoprotection from UVA by the sunscreen in inhibiting elastase activation, and also suggest the possibility of reducing photoaging.

Key words elastase; sagging; photoaging; sunscreen; ultraviolet

To elucidate the mechanism underlying skin wrinkle formation, one feature of photoaging, we recently performed a study in which rat hind limb (plantar) skin was short-term chronically irradiated with ultraviolet-B (UVB) at 1 MED or lower. That study showed that elastase activity was increased in the rat hind limb skin $^{1)}$ and changed the 3-dimensional structure of dermal elastic fibers, which bent the fibers responsible for skin elasticity ${ }^{2,3)}$ and thus decreased skin elasticity, forming wrinkles. ${ }^{1,4,5)}$ We also reported that this series of events could be inhibited by application of an elastase activity-inhibiting agent after UVB irradiation. ${ }^{1,6,7)}$ Inhibition of the UVB-induced increase in skin elastase activity prevented by the agent maintained the linearity of dermal elastic fibers and inhibited the decrease in skin elasticity, resulting in the inhibition of wrinkle formation. ${ }^{1,6,7)}$ This finding suggested that application of an elastase inhibitor immediately after UVB exposure would inhibit photoaging of the skin, mainly wrinkles.

It has been shown that chronic UVB irradiation of the dorsal skin forms wrinkles in hairless mice ${ }^{8,9)}$ and that chronic ultraviolet-A (UVA) irradiation forms mainly sags. ${ }^{8,9)}$ In animal models, chronic UVA irradiation increased skin elastase activity and destroyed the 3-dimensional structure of dermal elastic fibers, decreasing skin elasticity, similarly to UVB irradiation. ${ }^{5,9-11)}$ There have been many reports that the application of sunscreens before UV exposure inhibits the photoaging of skin in animal models and in humans. ${ }^{8,12-16)}$ Based on those findings, application of a sunscreen before UV exposure might be expected to inhibit an increase in skin elastase activity even when UVA is continuously irradiated. It is therefore important and interesting to investigate the ef- fects of chronic UVA irradiation on elastase activity in skin protected with sunscreens before irradiation in an animal model.

The purpose of this study was to investigate effects of the photoprotection from UVA by topically applied sunscreens on skin elastase activity and on skin properties.

\section{MATERIALS AND METHODS}

Animals Forty female HR/ICR hairless mice were used. This strain was derived by crossing 6 week old hairless mice (HR/HR) originally obtained from Nisseiken Corp (Tokyo, Japan) with the albino strain HaM/ICR. The HR/ICR strain represents a line maintained under clean conventional conditions in our laboratory by hairless brother/haired sister mating for several years. All experiments were performed with hairless female mice only, which had free access to food and water. They were housed in rooms where the lighting (without UVB emission) was automatically regulated on a $12 \mathrm{~h}$ light and dark cycle. The experimental protocol was approved by the Ethics Review Committee for Animal Experimentation of Kao Corp.

UVA Irradiation UVA irradiation was carried out as described previously. ${ }^{9,10)}$ Briefly, mice were irradiated using a bank of 12 Toshiba BL lamps with a glass filter $(0.5 \mathrm{~cm}$ thick) for UVA (peak of emission near $351 \mathrm{~nm}$, no emission below $320 \mathrm{~nm}$, the irradiance between 320 and $380 \mathrm{~nm}$ corresponded to $93 \%$ of the total amount of UVA). The distance from the lamps to the animals' backs was $35 \mathrm{~cm}$. The animals were exposed to a UVA dose of $22.3 \mathrm{~J} / \mathrm{cm}^{2} / \mathrm{d} 5$ times a week for 15 weeks. 
Samples Since irradiation with UVA alone (with complete blockage of UVB) is not possible due to the structure of the UVA irradiator (glass filter), the UVB sunscreen group was added to eliminate the influence of any slight leakage of UVB.

This experiment used common UVA and UVB sunscreen agents presently formulated in many cosmetic products on the market. ${ }^{17,18)}$ The vehicle (base: decamethylcyclopentasiloxane), a UVB sunscreen (3\% 2-ethylhexyl 4-methoxycinnamate + base), and a UVA\&UVB sunscreen (10\% silicone coated zinc oxide $+3 \%$ 2-ethylhexyl 4-methoxycinnamate+base) were tested. According to the Japan Cosmetic Industry Association standards, the Protection Factor of UVA (PFA) in the UVA sunscreen sample was approximately $6(\mathrm{PA}++)$, representing an $83.3 \%$ UVA protection efficacy, while the UVB sunscreen sample had an approximate Sun Protection Factor (SPF) of 20. Each sample $(30 \mu 1)$ was applied to the entire dorsal skin of each animal which was UVA irradiated $30 \mathrm{~min}$ later.

The mice were divided into the following 4 groups $(n=10$ in each group): (1) group 1, no treatment and no UVA irradiation (Untreated/UVA $(-)$ ); (2) group 2, irradiated with UVA after vehicle application (Vehicle/UVA $(+)$ ); (3) group 3, irradiated with UVA after application of the UVB sunscreen (UVB sunscreen/UVA $(+)$ ); (4) group 4, irradiated with UVA after application of the UVA\&UVB sunscreen (UVA\&UVB sunscreen/UVA $(+))$.

Evaluation of Skin Properties All procedures were performed 12 weeks after the course of UVA irradiation. Procedures to evaluate skin properties were performed under pentobarbital anesthesia.

1: Photography: A photograph of the dorsal skin of each mouse was taken using a Minolta $\alpha 707$ si camera with a macro 100 lens (Minolta Co., Ltd., Tokyo, Japan) system and a Nikon D1 digital camera (Nikon Corp., Tokyo, Japan), as detailed previously. ${ }^{10)}$

2: Evaluation of Recovery from Stretching: Pinch testing was carried out according to the method of Bryce and Bogdan. ${ }^{19)}$ The dorsal skin at the midline was picked up with the fingers as much as possible (to a degree that does not lift the animal into the air), and the pinch was subsequently released. The time (s) until the skin recovered to the original state was measured.

3: Measurement of Skin Color: For the measurement of skin color, a color meter (Model OFC-300A, Nippon Denshoku Industries Co., Ltd., Tokyo, Japan) was used. Measurements were performed 5 times on the midline $(1 \mathrm{~cm}$ above the root of the tail), and the $\mathrm{L}^{*}$ value (Luminance, $\mathrm{L}^{*}$ which represents the relative brightness from total darkness $\left(\mathrm{L}^{*}=0\right)$ to absolute white $\left(\mathrm{L}^{*}=100\right)$, the $\mathrm{a}^{*}$ value (red-green color axis) and the $b^{*}$ value (yellow-blue color axis) were calculated, and the mean values were obtained.

4: Measurement of Water Content of the Stratum Corneum: To measure the water content in the stratum corneum, a Skicon-200 ${ }^{\circledR}$ (IBS Co., Ltd., Hamamatsu, Japan) was used. Measurements were performed 5 times each on the right and left of the midline, and the mean values were obtained.

5: Measurement of Transepidermal Water Loss (TEWL): To measure cutaneous water evaporation, a Tewameter TM210 ${ }^{\circledR}$ (Courage+Khazaka electric GmbH, Köln, Ger- many) was used. Measurements were performed once each on the right and the left of the midline and the mean values were obtained.

6: Measurement of Skin Thickness: Skin thickness (epidermis \& dermis) was measured using a UX-02 ultrasonic diagnostic system (RION Co., Ltd., Tokyo, Japan). To evaluate skin thickness, B-mode ultrasonography was performed in a dynamic range of 60 with a gain of $8 \mathrm{~dB}$ at $30 \mathrm{MHz}$. All measured values are expressed as the median value of 5 recordings. ${ }^{9,10)}$ Ultrasonographic images obtained by the Bmode were printed (thermal recording paper for Mitsubishi video copy processor, SCT-K65H) using a video printer (SCT-P65 video copy processor, Mitsubishi Electric Corp., Tokyo, Japan). On ultrasonographic images, the thickness from the skin surface to the plane showing discontinuity of echogenicity, ${ }^{20)}$ which represents the border between the dermis and the subcutaneous tissue, was measured at 5 sites, and the mean values were calculated.

7: Measurement of Skin Elasticity: Skin elasticity was measured with a Cutometer Skin Elasticity Meter $575^{\circledR}$ (Courage+Khazaka electric GmbH, Köln, Germany), as detailed previously. ${ }^{9)}$ This instrument measures the elastic properties of skin, based on the principle of suction elongation, using an optical measuring unit described by Elsner et al. ${ }^{21)}$ Briefly, the time/strain mode was used with application of a $100 \mathrm{hPa}$ load for $1 \mathrm{~s}$ followed by $1 \mathrm{~s}$ of relaxation. The skin deformation was then plotted as a function of time. The parameters used were immediate distension (Ue), measured at $0.1 \mathrm{~s}$, delayed distension (Uv), immediate retraction (Ur), and final distension (Uf), as described by Agache et al. ${ }^{22)}$ Certain biologically relevant ratios of these parameters, e.g. Ur/Uf, the ratio between immediate retraction and total distension, represent the skin's ability to recover to its initial position after deformation. These parameters are found to be good indicators for evaluating skin elasticity. ${ }^{23)}$

Measurement of Elastase Activity Elastase activity in the mouse dorsal skin was measured after 15 weeks of irradiation with UVA using the synthetic substrate STANA $(N$ succinyl-tri-alanyl- $p$-nitroanilide, Peptide Institute, Osaka, Japan), as described by Nakagawa et al. ${ }^{24)}$ In brief, hairless mouse skin was biopsied and after removing the subcutaneous tissue, it was homogenized and solubilized in $0.1 \%$ Triton-X 100, 0.2 м Tris- $\mathrm{HCl}(\mathrm{pH} 8.0)$ buffer, followed by ultrasonication and by centrifugation $(2000 \boldsymbol{g} \times 20 \mathrm{~min})$ to obtain supernatants for enzyme assay. To measure the elastase activity, $100 \mu \mathrm{l}$ of the enzyme solution was dispensed into 96-well plates which were pre-incubated for $15 \mathrm{~min}$ at $37^{\circ} \mathrm{C}$. After the addition of $2 \mu 162.5 \mathrm{~mm}$ STANA, further incubation was performed for $1 \mathrm{~h}$ at $37^{\circ} \mathrm{C}$. The release of $p$-nitroaniline was measured by the absorbance at $405 \mathrm{~nm}$ and the enzymatic activity is expressed as unit $/ \mathrm{cm}^{2}$ skin representing the activity that releases $1 \mathrm{nmol}$ nitroaniline/h.

Statistics Results are expressed as means \pm standard deviation, as noted in the Figures and Tables. Differences between means were determined for statistical significance using the one way ANOVA followed by Tukey's post hoc multiple comparison test.

\section{RESULTS}

Effect of Sunscreens on Sagging Figure 1A shows pho- 
tos of dorsal skins approximately $1 \mathrm{~s}$ after stretching with or without chronic UVA irradiation for 12 weeks. Regarding the ability of sags to recover as examined by the pinch test, it was significantly decreased in the Vehicle/UVA $(+)$ group and in the UVB sunscreen/UVA $(+)$ group, compared to the Untreated/UVA $(-)$ group. The decreased recovery was significantly inhibited in the UVA\&UVB sunscreen/UVA $(+)$ group compared to the Vehicle/UVA $(+)$ and the UVB sunscreen/UVA $(+)$ groups, and the level was similar to that in the Untreated/UVA( $(-)$ group (Fig. 1B).

Effect of Sunscreens on Skin Color Table 1 shows the measurements of skin color in the various groups. UVA irradiation significantly increased the $\mathrm{L}^{*}$ value, which represents brightness, in the Vehicle/UVA $(+)$ and the UVB sun-
screen/UVA $(+)$ groups, compared to the Untreated/UVA $(-)$ group. However, that increase was significantly inhibited in the UVA\&UVB sunscreen/UVA $(+)$ group. UVA irradiation also significantly increased the $b^{*}$ value (yellowness) in the Vehicle/UVA $(+)$ and the UVB sunscreen/UVA $(+)$ groups, but not in the UVA\&UVB sunscreen/UVA $(+)$ group compared to the Untreated/UVA $(-)$ group. In contrast, there was no significant change in the $\mathrm{a}^{*}$ value (redness) in any of the groups.

Effect of Sunscreens on Stratum Corneum Function Regarding changes in water content in the stratum corneum, UVA irradiation significantly decreased the water content in the Vehicle/UVA $(+)$ and in the UVB sunscreen/UVA $(+)$ groups, compared to the Untreated/UVA $(-)$ group (Fig. 2).

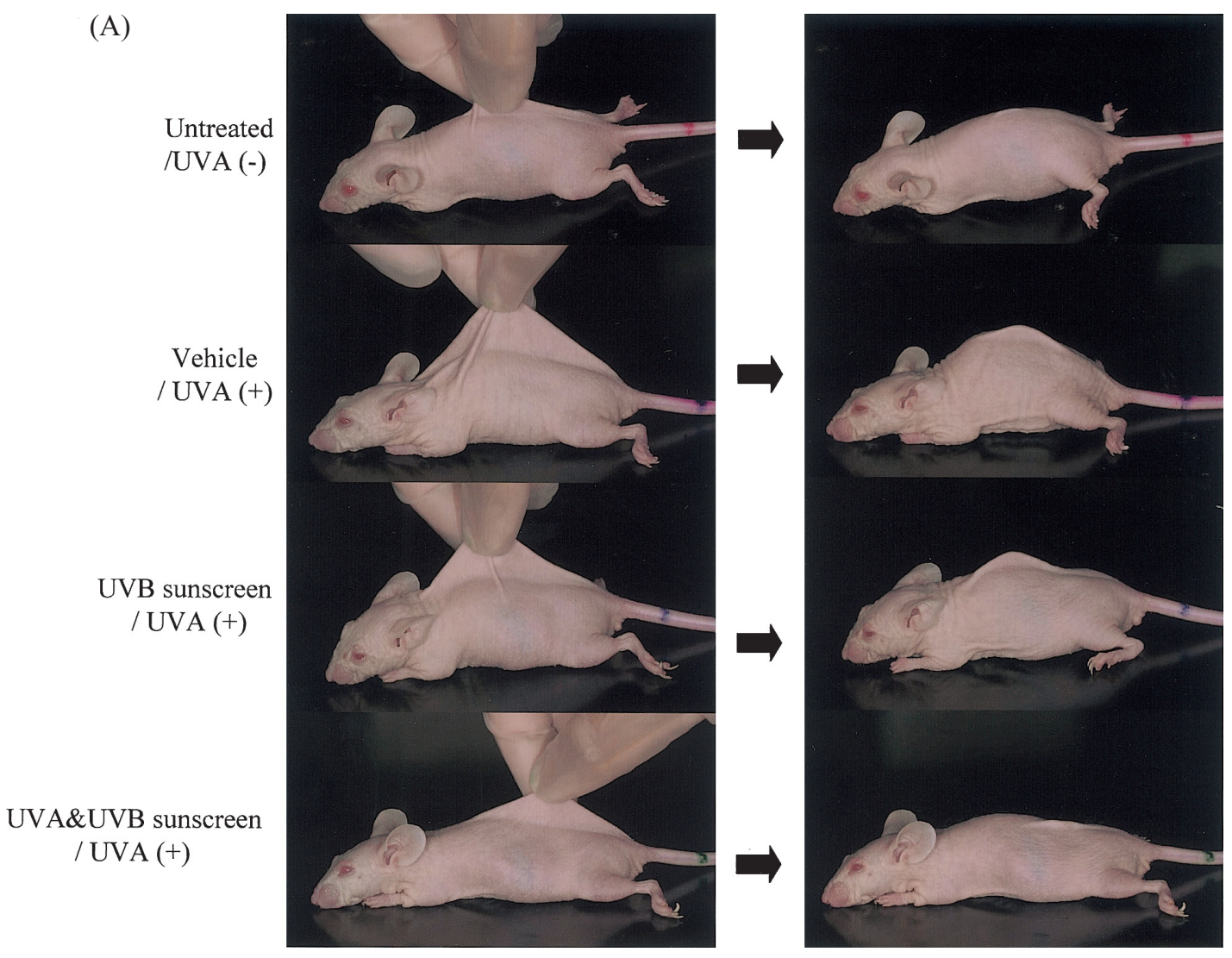

(B)

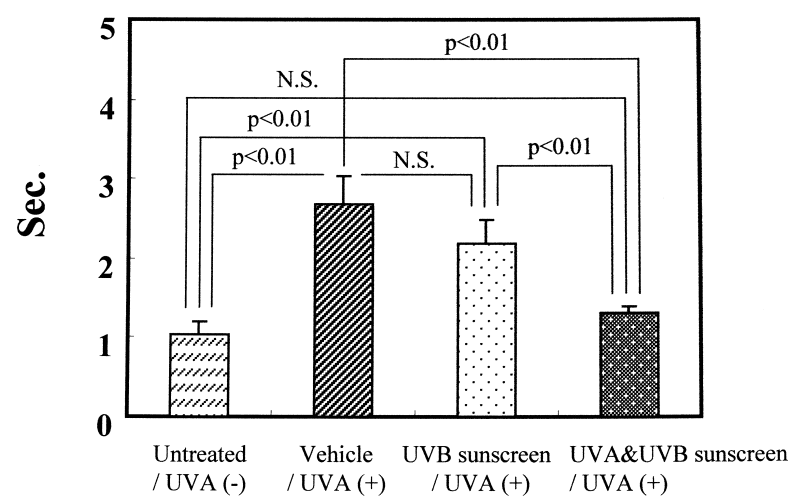

Fig. 1. Evaluation of Sagging by Pinch Testing

(A) Photographs of pinch testing carried out according to the method of Bryce and Bogdan. ${ }^{19)}$ Left: Mouse dorsal skin at the midline was picked up with the fingers as much as possible without lifting the mouse. Right: Photograph $1 \mathrm{~s}$ after the skin was released. (B) Recovery time in the pinch test in groups with or without UVA exposure (5 times weekly for 12 weeks). Data represent means \pm S.D. Differences between means were determined for statistical significance using the one way ANOVA followed by Tukey's post-hoc multiple comparison test. 
Table 1. Skin Color of Hairless Mouse with or without UVA Irradiation

\begin{tabular}{|c|c|c|c|}
\hline Group & $\mathrm{L}^{*}$ value & $\mathrm{a}^{*}$ value & $\mathrm{b}^{*}$ value \\
\hline Untreated/UVA(-) & $48.98 \pm 1.61$ & $1.13 \pm 0.38$ & $-0.97 \pm 0.42$ \\
\hline Vehicle/UVA $(+)$ & $52.18 \pm 1.64^{\S}$ & $0.80 \pm 0.38$ & $0.35 \pm 1.00^{\S}$ \\
\hline UVB sunscreen/UVA(+) & $52.46 \pm 1.24^{\S}$ & $1.01 \pm 0.19$ & $0.23 \pm 0.93^{\S}$ \\
\hline UVA\&UVB sunscreen/UVA(+) & $48.28 \pm 1.30^{\#, \text { ศศ }}$ & $1.17 \pm 0.32$ & $-0.98 \pm 0.72^{\#}$ \\
\hline
\end{tabular}

Data represent mean \pm S.D. $s p<0.05(v s$. Untreated/UVA $(-))$. \# $p<0.05(v s$. Vehicle/UVA $(+))$. $\uparrow p<0.01(v s$. UVB sunscreen/UVA $(+))$. Differences between means were determined for statistical significance using the one way ANOVA followed by Tukey's post-hoc multiple comparison test.

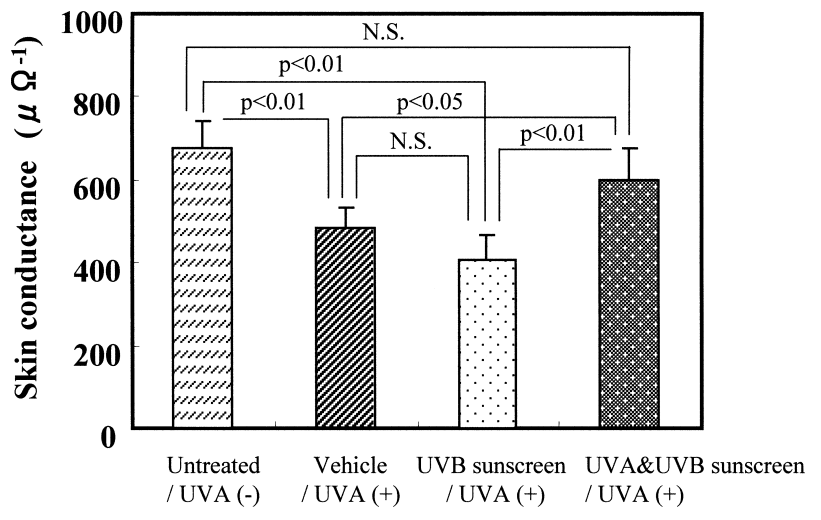

Fig. 2. Changes in Water Content of the Stratum Corneum after UVA Irradiation (5 Times Weekly for 12 Weeks)

Data represent means \pm S.D. Differences between means were determined for statistical significance using the one way ANOVA followed by Tukey's post-hoc multiple comparison test.

However, that decrease was significantly inhibited in the UVA\&UVB sunscreen/UVA(+) group, which had a level similar to the Untreated/UVA(-) group.

In contrast, no significant change was noted in TEWL in any of the groups (Fig. 3).

Effect of Sunscreens on Skin Thickness UVA irradiation significantly increased the skin thickness in the Vehicle/UVA $(+)$ and the UVB sunscreen/UVA $(+)$ groups, compared to the Untreated/UVA $(-)$ group (Fig. 4). However, that increase was significantly inhibited in the UVA\&UVB sunscreen/UVA $(+)$ group which was similar to the Untreated/ $\mathrm{UVA}(-)$ group.

\section{Effect of Sunscreens on Skin Elasticity}

Table 2 shows the results of the major parameters, Ue, $\mathrm{Uf}$, and $\mathrm{Ur} / \mathrm{Uf}$ in the various groups. UVA irradiation significantly decreased Ue (immediate distension) in the Vehicle/UVA $(+)$ and the UVB sunscreen/UVA $(+)$ groups, compared to the Untreated/UVA $(-)$ group. Ue was also significantly decreased in the UVA\&UVB sunscreen/UVA $(+)$ group, compared to the Untreated/UVA(-) group, but the decrease was significantly inhibited compared to the Vehicle/UVA $(+)$ and the UVB sunscreen/UVA(+) groups.

UVA irradiation significantly decreased Uf (final distension) and Ur/Uf (the biologically relevant ratio) in the Vehicle/UVA $(+)$ and the UVB sunscreen/UVA $(+)$ groups, compared to the Untreated/UVA(-) group. However, those decreases were significantly inhibited in the UVA\&UVB sunscreen/UVA(+) group which had levels similar to the Untreated/UVA $(-)$ group.

Effect of Sunscreens on Skin Elastase Activity Skin elastase activity with or without 15 weeks of UVA irradiation is shown in Fig. 5. UVA irradiation significantly increased

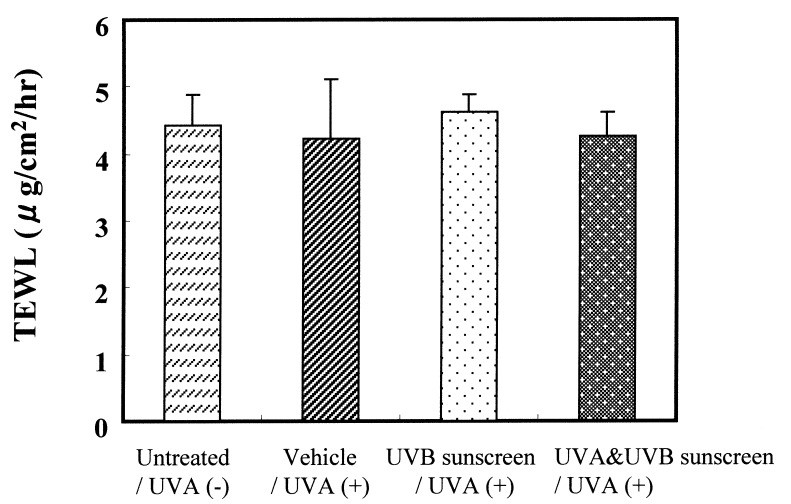

Fig. 3. Changes of TEWL after UVA Irradiation (5 Times Weekly for 12 Weeks)

Data represent means \pm S.D. Differences between means were determined for statistical significance using the one way ANOVA followed by Tukey's post-hoc multiple comparison test. Statistically significant differences were not recognized among any of the groups.

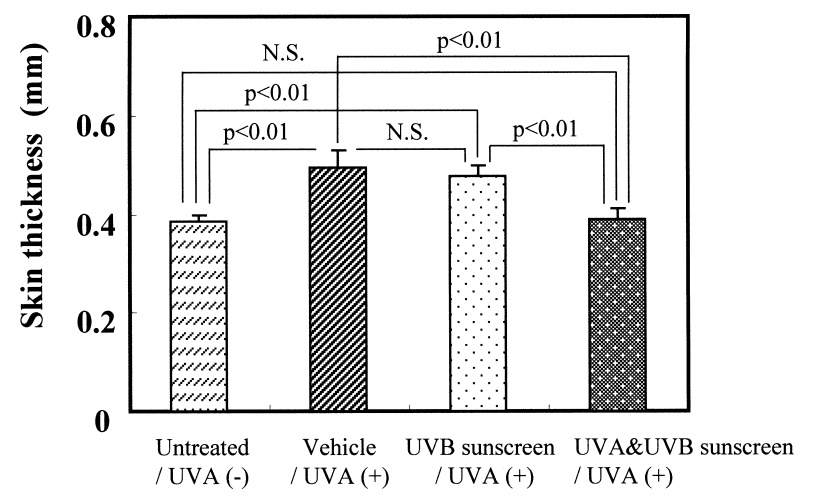

Fig. 4. Changes in Skin Thickness after UVA Irradiation (5 Times Weekly for 12 weeks)

Data represent means \pm S.D. Differences between means were determined for statistical significance using the one way ANOVA followed by Tukey's post-hoc multiple comparison test.

the elastase activity in the Vehicle/UVA(+) and the UVB sunscreen/UVA $(+)$ groups, compared to the Untreated/ UVA(-) group. Elastase activity was also significantly increased in the UVA\&UVB sunscreen/UVA $(+)$ group compared to the Untreated/UVA $(-)$ group, but the increase was significantly inhibited compared to the Vehicle/UVA $(+)$ group.

\section{DISCUSSION}

In this study, we verified effects of the topical photoprotection from UVA by the sunscreens on skin elastase activity and on skin properties, using the dorsal skins of hairless mice. Compared to hairless mice treated with vehicle only 
Table 2. Skin Elasticity of Hairless Mouse with or without UVA Irradiation

\begin{tabular}{lll}
\hline \hline Group & Ue & Uf \\
\hline Untreated/UVA(-) & $0.317 \pm 0.06$ & $0.174 \pm 0.04$ \\
Vehicle/UVA(+) & $0.199 \pm 0.02^{\S \S}$ & $0.087 \pm 0.01^{\S \S}$ \\
UVB sunscreen/UVA(+) & $0.209 \pm 0.02^{\S \S}$ & $0.086 \pm 0.01^{\S \S}$ \\
UVA\&UVB sunscreen/UVA(+) & $0.260 \pm 0.01^{\S, \# \text {, , }}$ & $0.145 \pm 0.01^{\#, \text {, }}$ \\
\hline
\end{tabular}

Data represent mean \pm S.D. $s \S, \$ p<0.01,0.05(v s$. Untreated/UVA $(-))$. \# $p<0.01(v s$. Vehicle/UVA(+)). $\uparrow, \uparrow p<0.01,0.05(v s$. UVB sunscreen/UVA $(+))$. Differences between means were determined for statistical significance using the one way ANOVA followed by Tukey's post-hoc multiple comparison test.

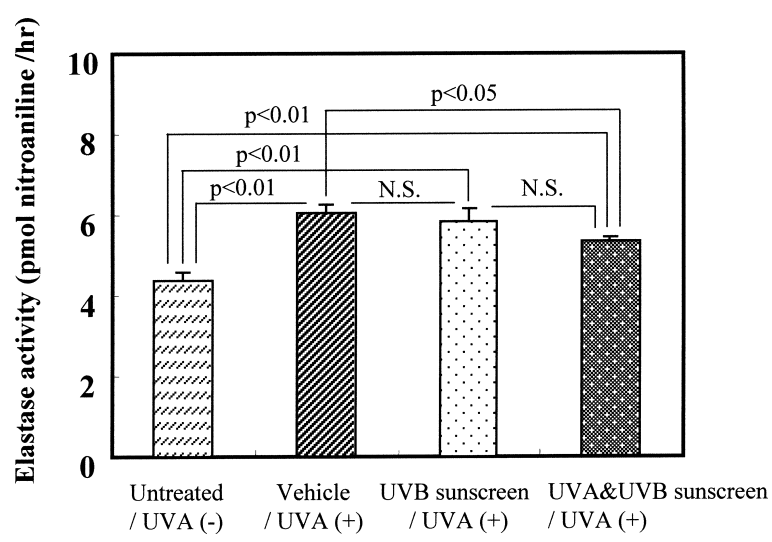

Fig. 5. Effects of UV Protective Materials with UVA on Elastase Activity

Elastase activity was measured using STANA as a substrate in skin extracts from control and from UVA-exposed mice as described in the Materials and Methods. Data represent means \pm S.D. Differences between means were determined for statistical significance using the one way ANOVA followed by Tukey's post-hoc multiple comparison test.

before UVA irradiation, sagging of skin, increases in brightness and yellowing, decrease in water content of the stratum corneum, increase in skin thickness and decrease in skin elasticity, were inhibited in the UVA\&UVB sunscreen group. Application of UVA\&UVB sunscreen before UVA irradiation also inhibited the UVA-induced increase in skin elastase activity after 15 weeks of UVA irradiation, compared to the vehicle group.

Bissett et al. reported that irradiation with UVA 3 times a week for 33 weeks at $7.5 \mathrm{~J} / \mathrm{cm}^{2} / \mathrm{d}$ caused sags after the 28 th week in hairless mouse skin, that there was a significant increase in skin thickness, and that skin color was decreased. ${ }^{8)}$ Takema and Imokawa reported that chronic UVA irradiation at $22.3 \mathrm{~J} / \mathrm{cm}^{2} / \mathrm{d}$ for 8 weeks significantly decreased skin elasticity in hairless mice compared to unirradiated control mice. ${ }^{10)}$ It has also recently been shown that irradiation of hairless mice with UVA at $35 \mathrm{~J} / \mathrm{cm}^{2} / \mathrm{d} 3$ times a week for 52 weeks or at $22.3 \mathrm{~J} / \mathrm{cm}^{2} / \mathrm{d} 5$ times per week for 14 weeks increased skin elastase activity. ${ }^{9,11)}$ Comparison of the Untreated/UVA $(-)$ and the Vehicle/UVA $(+)$ groups in our study showed that sagging of the skin, decreases in skin elasticity, and increases in skin thickness, were consistent with those previous reports. ${ }^{8-11)}$ In addition, skin properties in the group exposed to chronic UVA irradiation and treated with the UVB sunscreen alone were similar to those in the Vehicle/UVA $(+)$ group. The UVB sunscreen/UVA $(+)$ group was used to determine the effects of UVA alone by protecting the skin from UVB, which a glass filter cannot totally block. This result showed that the effect of UVB irradiation which might have leaked through the glass filter was negligible in this study.
In the group treated with the UVA\&UVB sunscreen prior to chronic UVA irradiation, a photoaging-inhibiting effect was noted compared to the Vehicle/UVA $(+)$ group. However, compared to the Untreated/UVA $(-)$ group, the major parameter of skin elasticity, Ue (immediate distension), was significantly decreased in the UVA\&UVB sunscreen/ $\mathrm{UVA}(+)$ group, showing that skin elastase activity was significantly increased. These findings suggest that application of the UVA\&UVB sunscreen before UVA irradiation alone could not completely inhibit photoaging induced by UVA irradiation for 15 weeks. We presume that the UVA which penetrated the sunscreen, which theoretically protects the skin against $83 \%$ of the UVA, might still induce some photoaging. It has been reported that elastase activity in the dorsal skin increases with age in hairless mice, and that chronic irradiation with UVA and UVB increases the activity earlier than aging-related changes. ${ }^{911)}$ We previously reported that application of an elastase activity-inhibiting agent after UVB irradiation reduces the increase in elastase activity, maintains the linearity of dermal elastic fibers, and inhibits the decrease in skin elasticity, which results in the inhibition of wrinkle formation. ${ }^{1,6,7)}$ Chronic UVA irradiation causes mainly sagging of the skin. ${ }^{8,9)}$ In the skin, elastase activity is increased, and elastic fibers lose their 3-dimensional structure, thereby decreasing skin elasticity. ${ }^{5,9-11)}$ This phenomenon is similar to that of UVB irradiated skin, which suggests that the application of an elastase inhibitor immediately after UVA irradiation may prevent skin sagging. Thus, to prevent skin aging, the concomitant use of a material that reduces or recovers damage after UV exposure, that is, agents that inhibit elastase activity, ${ }^{1,6,7)}$ in addition to an effective sunscreen, may be more effective in inhibiting the photoaging of skin.

The TEWL level was similar in the Untreated/UVA(-) and the Vehicle/UVA $(+)$ groups, and no increase in TEWL was induced by chronic UVA irradiation. It has been reported that chronic UVB irradiation induces an increase in TEWL in the dorsal skin of hairless mice, ${ }^{8,25)}$ but there has been no report of an increase in TEWL in the dorsal skin induced by chronic UVA irradiation in hairless mice. Bissett et al. reported that when skin was UVA irradiated for 33 weeks, the TEWL was significantly increased in the 31 st week compared to the level before irradiation, but it was not significantly different from TEWL in the group without UVA irradiation at the same age. ${ }^{8}$ UVA may increase TEWL only when it is irradiated at a very high dose and/or for a prolonged period.

The sum of these results shows that the topical photoprotection from UVA by the sunscreen inhibits elastase activity and is, therefore, considered to be an effective treatment for relieving symptoms of photoaging, mainly in the form of sags. However, the sunscreen alone is not completely effec- 
tive, which suggests that a combination of a sunscreen and an elastase inhibitor would be more efficient.

Ackowledgements We would like to thank Mr. Makoto Inoue from the Performance Chemicals Research Laboratories, Kao Corporation, for technical support of this study.

\section{REFERENCES}

1) Tsukahara K., Takema Y., Moriwaki S., Tsuji N., Suzuki Y., Fujimura T., Imokawa G., J. Invest. Dermatol., 117, 671-677 (2001).

2) Uitto J., Dermatol. Clin., 4, 433-446 (1986).

3) Oxlund H., Manschot J., Viidik A., J. Biomech., 21, 213-218 (1988).

4) Imayama S., Nakamura K., Takeuchi M., Hori Y., Takema Y., Sakaino Y., Imokawa G., J. Dermatol. Sci., 7, 32-38 (1994).

5) Imokawa G., Takema Y., Yorimoto Y., Tsukahara K., Kawai M., Imayama S., J. Invest. Dermatol., 105, 254-258 (1995).

6) Tsukahara K., Moriwaki S., Fujimura T., Takema Y., Biol. Pharm. Bull., 24, 998-1003 (2001).

7) Tsukahara K., Nakagawa H., Moriwaki S., Takema Y., Fujimura T., Imokawa G., Int. J. Dermatol., (2005) in press.

8) Bissett D. L., Hannon D. P., Orr T. V., Photochem. Photobiol., 46, 367-378 (1987).

9) Tsukahara K., Nakagawa H., Moriwaki S., Kakuo S., Ohuchi A. Takema Y., Imokawa G., Br. J. Dermatol., 151, 984-994 (2004).

10) Takema Y., Imokawa G., Dermatology, 196, 397-400 (1998).

11) Labat-Robert J., Fourtanier A., Boyer-Lafargue B., Robert L., J. Pho- tochem. Photobiol. B., 57, 113-118 (2000).

12) Kligman L. H., Akin F. J., Kligman A. M., J. Invest. Dermatol., 78, $181-189$ (1982)

13) Kligman L. H., Akin F. J., Kligman A. M., J. Invest. Dermatol., 81, 98-102 (1983).

14) Bissett D. L., McBride J. F., Hannon D. P., Patrick L. F., J. Photochem. Photobiol. B., 9, 323-334 (1991).

15) Fourtanier A., Labat-Robert J., Kern P., Berrebi C., Gracia A. M., Boyer B., Photochem. Photobiol., 55, 549-560 (1992).

16) Boyd A. S., Naylor M., Cameron G. S., Pearse A. D., Gaskell S. A., Neldner K. H., J. Am. Acad. Dermatol., 33, 941—946 (1995).

17) Sakurai T., Saito K., Fragrance J., 29, 79-83 (1999)

18) Sugawara S., Inomata Y., Fragrance J., 32, 72-80 (2004).

19) Bryce G. F., Bogdan N. J., J. Invest. Dermatol., 96, 584 (1991) Abstract.

20) Tsukahara K., Takema Y., Moriwaki S., Fujimura T., Kitahara T., Imokawa G., Dermatology, 200, 303-307 (2000).

21) Elsner P., Wilhelm D., Maibach H. I., Br. J. Dermatol., 122, 607-614 (1990).

22) Agache P. G., Monneur C., Leveque J. L., De Rigal J., Arch. Dermatol. Res., 269, 221-232 (1980).

23) Takema Y., Yorimoto Y., Kawai M., Imokawa G., Br. J. Dermatol., 131, 641-648 (1994).

24) Nakagawa K., Tsuji T., Kadoya A., Hamada T., Hifu (Skin Res.) Jpn., 29, 793-797 (1989).

25) Haratake A., Uchida Y., Schmuth M., Tanno O., Yasuda R., Epstein J. H., Elias P. M., Holleran W. M., J. Invest. Dermatol., 108, 769-775 (1997). 